\title{
Imbalance of heterologous protein folding and disulfide bond formation rates yields runaway oxidative stress
}

Keith EJ Tyo ${ }^{1,2}$, Zihe Liu', Dina Petranovic ${ }^{1}$ and Jens Nielsen ${ }^{1 *}$

\begin{abstract}
Background: The protein secretory pathway must process a wide assortment of native proteins for eukaryotic cells to function. As well, recombinant protein secretion is used extensively to produce many biologics and industrial enzymes. Therefore, secretory pathway dysfunction can be highly detrimental to the cell and can drastically inhibit product titers in biochemical production. Because the secretory pathway is a highly-integrated, multi-organelle system, dysfunction can happen at many levels and dissecting the root cause can be challenging. In this study, we apply a systems biology approach to analyze secretory pathway dysfunctions resulting from heterologous production of a small protein (insulin precursor) or a larger protein ( $\alpha$-amylase).

Results: HAC1-dependent and independent dysfunctions and cellular responses were apparent across multiple datasets. In particular, processes involving (a) degradation of protein/recycling amino acids, (b) overall transcription/ translation repression, and (c) oxidative stress were broadly associated with secretory stress.

Conclusions: Apparent runaway oxidative stress due to radical production observed here and elsewhere can be explained by a futile cycle of disulfide formation and breaking that consumes reduced glutathione and produces reactive oxygen species. The futile cycle is dominating when protein folding rates are low relative to disulfide bond formation rates. While not strictly conclusive with the present data, this insight does provide a molecular interpretation to an, until now, largely empirical understanding of optimizing heterologous protein secretion. This molecular insight has direct implications on engineering a broad range of recombinant proteins for secretion and provides potential hypotheses for the root causes of several secretory-associated diseases.
\end{abstract}

Keywords: Protein secretion, unfolded protein response, HAC1, protein production, oxidative stress

\section{Background}

The protein secretory pathway is an extensive process in eukaryal cells, as it is responsible for processing approximately one-third of all proteins. Substantial cellular resources are therefore utilized to maintain this pathway's functions, and stressed conditions in the secretory pathway have consequences for the whole cell [1]. Distress in secretory pathway organelles has been implicated as the molecular basis for several diseases, for example, $\beta$ cell apoptosis in diabetes, cystic fibrosis, and prion-related disease, among others [2]. In biotechnology, efficient

\footnotetext{
* Correspondence: nielsenj@chalmers.se

'Department of Chemical and Biological Engineering, Chalmers University of Technology, Kemivägen 10, SE-41296 Göteborg, Sweden

Full list of author information is available at the end of the article
}

secretion of useful recombinant proteins in yeast and fungi is a key industrial objective with applications in enzyme production required for the production of biofuels, detergents, fabrics, food, and biologics, such as imunoglobulins, hormones, and vaccines. Significant effort has gone into engineering yeast for increasing protein secretion [3]. Strategies, such as changing environmental parameters (for example, temperature, media composition) [4] or altering genetics, can increase secretion for some proteins, but they rarely represent generic solutions for improving protein secretion [5,6]. The lack of a single engineering strategy that improves protein secretion across the board implies that there are several possible bottlenecks in the secretory pathway, and different proteins may be constrained in different ways. There is therefore a
C Biomed Central

() 2012 Tyo et al; licensee BioMed Central Ltd. This is an Open Access article distributed under the terms of the Creative Commons Attribution License (http://creativecommons.org/licenses/by/2.0), which permits unrestricted use, distribution, and reproduction in any medium, provided the original work is properly cited. 
requirement for more fundamental insight into this complex pathway that involves a very large number of components.

In yeast, the secretory pathway is a multi-organelle system that is responsible for trafficking proteins to the extracellular space, cell membrane, or vacuole [7]. During this transit, multiple processes must be coordinated, including folding, specific proteolytic cleavage, glycosylation, and disulfide bond formation, all with a layer of quality control at key check points. The pathway requires substantial cellular resources to perform these tasks, such as glycans, electron acceptors, electron donors, and ATP. In the ER, the nascent peptide is folded into its native structure while disulfide bonds are formed. The rate of protein folding is dependent upon the complexity of the protein to be folded, the availability of chaperones to assist folding, and ATP used by the chaperones [1]. Proteins that are slow to fold or terminally misfolded proteins are removed from the ER via the ER-associated degradation (ERAD) pathway [8]. Disulfide bond formation requires the removal of electrons from cysteine thiols via protein disulfide isomerase (PDI) and Erolp to the final electron acceptor, typically oxygen $[9,10]$. This process produces reactive oxygen species (ROS) in stoichiometric amounts to the number of disulfide bonds formed [11]. Disulfide bond formation is random, and incorrect bond pairs must be exchanged for native bonds via PDI-based processes [12]. In addition, reduced glutathione (GSH) acts as a buffer for the redox state of the ER [13]. A more detailed description of oxidative protein folding can be found in the reviews by Sevier et al. and Chakravarthi et al. [14,15].

The secretory pathway must adjust the chaperone capacity, oxidizing equivalents, ATP, glycan, and other metabolic requirements, as well as trafficking patterns, based on the portfolio of proteins that need to be expressed at a given time, and the resources required to process that set of proteins. In yeast, the unfolded protein response (UPR) is one transcriptional mechanism that adjusts secretory resources and controls to handle overload of the folding machinery in the ER [16]. In the UPR, accumulation of unfolded proteins in the ER signals a pathway that results in translation of Hac1p, a transcription factor (TF) known to activate or repress over 100 genes, including many ER-associated proteins such as Kar2p, Pdi1p, and Ero1p [17].

In this study, we identified biological mechanisms which alter the secretory pathway in response to secretion of recombinant proteins with different properties (size, number of disulfide bonds, and glycans) in a Hac1p-dependent and independent manner. The secretory pathway was perturbed by secreting a small protein, human insulin precursor (IP), or a comparatively larger protein, $\alpha$-amylase, in wild-type (WT) and $\Delta h a c 1$
Saccharomyces cerevisiae. These proteins were chosen because the two proteins elicit different behavior in the secretory pathway. These differences will arise because $\alpha$-amylase is a relatively larger (and likely more difficult to fold), has an odd number of cysteines (which may complicate disulfide isomerization) and has glycosylation, compared to insulin which is small, has even number of cysteines, and is not glycosylated. As well, $\alpha$-amylase has one more disulfide bond than IP. To identify biological mechanisms, we characterized changes in physiological properties (specific growth rate, carbon utilization efficiency, and recombinant protein secretion), TF activity (as inferred from transcriptome analysis) and metabolic demand (as inferred by changes in metabolic flux diversion). Through this, we identified the following biological processes: amino acids recycling from degraded proteins, trans-Golgi network (TGN) sorting changes, overall expression repression, and oxidative stress. Motivated by secretory-related oxidative stress observations, we present a model for disulfide bond formation and electron transfer in the ER which takes into account thermodynamic irreversibilities caused by differences in electron affinity. The proposed model explains the non-stoichiometric ROS formation that we observed that results from disulfide bond formation and causes oxidative stress under folding-stress conditions. If proven by genetic and biochemical results, the futile cycle model yields insight into a fundamental problem in secretory stress and reveals new avenues to reduce oxidative stress and increase productivity in industrial protein production.

\section{Results \\ Protein size and Hac1p activity affect protein secretion quantity and cell growth}

Yeast strains were constructed that produce and secrete (a) IP or (b) $\alpha$-amylase and were compared to yeast strains containing (c) an empty vector in both wild-type and $H A C 1$ deletion backgrounds. IP and $\alpha$-amylase were chosen because they are very different types of proteins to secrete. IP is 51 amino acids in length, with six cysteines forming three disulfide bonds, and no glycosylation. $\alpha$-amylase is 478 amino acid in length, with nine cysteines forming only four disulfide bonds and one glycosylation. The odd number of cysteines in $\alpha$-amylase complicates disulfide pairing, as the random isomerization process may incorporate the cysteine that should not be incorporated into a disulfide bond. Both proteins were targeted for secretion using a YAP3 pre sequence (21 amino acids, cleaved off in the ER) and a rationally designed pro sequence (TA57, 42 amino acids, no glycosylation or disulfides) were cloned behind a $T D H 3$ promoter in a high copy 2 micron plasmid [18]. $\alpha$-amylase was expressed using the same plasmid, promoter, and leader sequences. These strains are named WN (WT 
with empty vector), WI (WT secreting IP), WA (WT secreting $\alpha$-amylase), $\mathrm{dN}$ ( $\Delta$ hac1 with empty vector), $\mathrm{dI}$ ( $\Delta h a c 1$ secreting IP), and dA ( hac1 secreting $\alpha$-amylase). Strains were characterized in batch fermentation to understand the effects on cell physiology.

The cellular burden induced by (a) synthesizing and secreting IP and $\alpha$-amylase and (b) deleting the key TF for the UPR, Hac1p, substantially affected the cells. Protein titers in WT strain were $9 \mathrm{mg} / \mathrm{L}$ and $20 \mathrm{mg} / \mathrm{L}$, for IP and $\alpha$-amylase, respectively (Figure 1a). On a per biomass basis, this is approximately half the insulin produced, and one-third the $\alpha$-amylase reported for rich media $[19,20]$. Rich media appears to be favorable for heterologous protein production, but may present complications in downstream separations. Comparing the small and larger proteins, $\alpha$-amylase was secreted in higher levels on a mass basis, but six-fold more insulin molecules were secreted $(1.52 \mu \mathrm{M}$ IP in WI compared to $0.26 \mu \mathrm{M} \alpha$-amylase in WA). 4 hac1 strains secreted significantly less protein than WT, confirming that Hac1p is important for efficient secretion (Figure 1a) [5].

Reduced specific growth rates imply impairment of cellular processes (Figure 1b). In WT yeast, IP production did not affect growth; however, $\alpha$-amylase production reduced growth by $25 \%$. This, combined with the differences in protein titers, implies that $\alpha$-amylase is more challenging to fold and secrete than IP. In the $\Delta$ hac1 background, recombinant protein strains $\mathrm{dI}$ and $\mathrm{dA}$ had approximately $20 \%$ lower growth rates compared to $\mathrm{dN}$. This growth reduction occurs despite no change in specific glucose uptake rate (Additional file 1, Tables S1 and S2) pointing toward higher energy requirements to maintain homeostasis in $\Delta$ hac1 while trying to secrete recombinant proteins. $\Delta$ hac1 strains had overall lower final cell densities. hac1 strains produced more glycerol than WT strains implying impaired oxidative processes in the $\Delta$ hac1 strains (Additional file 2).

\section{Secretory stress shifts metabolism to increase oxygen and ATP requirements}

The physiological changes due to the secretory perturbations affect the distribution of resources through the metabolic network. The glucose uptake and range of products produced were altered by the protein production conditions (Table 1). Changes in the underlying metabolic network were estimated by flux balance analysis (FBA) using a yeast central carbon metabolism model, constrained by measured extracellular fluxes (Additional file 1, Tables S1 and S2, Additional files 3 and 4) [21]. Figure 2a shows a metabolic map of central carbon metabolism for each of the six conditions based on the exchange fluxes in Table 1 and the FBA analysis. The shift in metabolic fluxes were correlated with changes in redox requirements. As expected, the catabolic functions of the TCA cycle was predicted to have very low activity due to glucose repression [22]. Figure $2 \mathrm{~b}$ shows that the oxygen uptake was twice as high in the strains that were growth inhibited (for example, WA, dI, dA) than those that were not. This increased oxygen uptake was not used for oxidative phosphorylation, as the biomass yields on glucose were lower in WA, dI, and dA, and it may therefore be a result of increased oxidation in connection with formation of disulfide bonds.

Figure $2 \mathrm{c}$ shows that the maintenance ATP consumption is increased in WA, dI, and dA according to FBA calculations. In WT background, WI did not consume a detectable increase in ATP, likely because IP is short

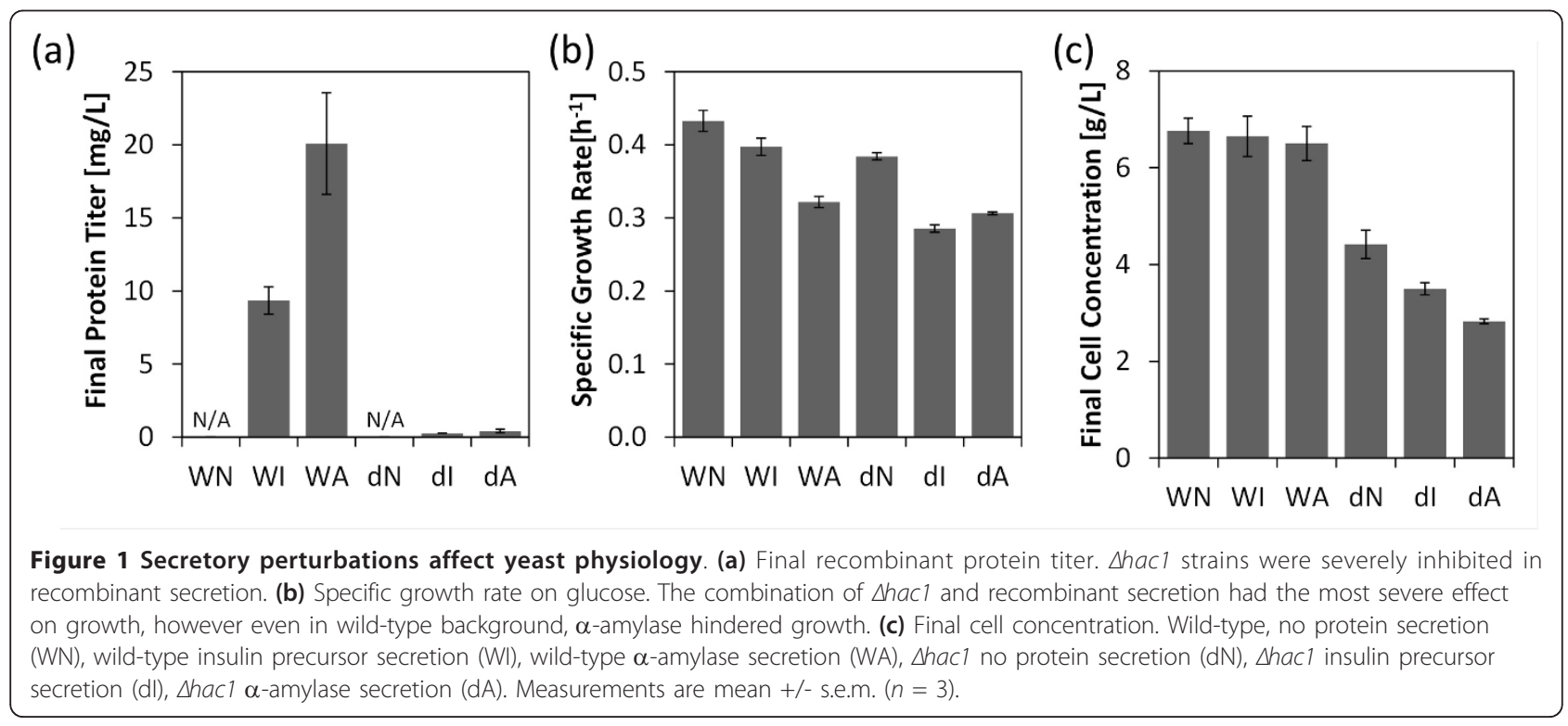


Table 1 Physiological parameters of recombinant protein secretion strains ${ }^{\mathrm{a}}$

\begin{tabular}{llllllll}
\hline Strains $^{\mathbf{b}}$ & $\mathbf{M}_{\max }\left[\mathrm{h}^{-1}\right]$ & $\mathrm{Y}_{\mathrm{SX}}$ & $\mathrm{Y}_{\mathrm{SE}}$ & $\mathrm{Y}_{\mathrm{SG}}$ & $\mathrm{Y}_{\mathrm{SA}}$ & $\mathrm{Y}_{\mathrm{SCO} 2}$ & Carbon balance \\
\hline $\mathrm{WN}$ & $0.43+/-0.014$ & $0.14+/-0.001$ & $0.32+/-0.041$ & $0.067+/-0.009$ & $0.048+/-0.0005$ & $0.30+/-0.019$ & 0.89 \\
WI & $0.40+/-0.012$ & $0.13+/-0.002$ & $0.35+/-0.029$ & $0.055+/-0.005$ & $0.056+/-0.0047$ & $0.30+/-0.014$ & 0.92 \\
WA & $0.32+/-0.007$ & $0.11+/-0.003$ & $0.31+/-0.006$ & $0.060+/-0.007$ & $0.049+/-0.0023$ & $0.30+/-0.002$ & 0.84 \\
$\mathrm{dN}$ & $0.38+/-0.005$ & $0.13+/-0.004$ & $0.37+/-0.025$ & $0.046+/-0.003$ & $0.035+/-0.0046$ & $0.29+/-0.020$ & 0.91 \\
$\mathrm{dl}$ & $0.29+/-0.005$ & $0.08+/-0.006$ & $0.32+/-0.017$ & $0.081+/-0.001$ & $0.046+/-0.0011$ & $0.31+/-0.007$ & 0.84 \\
$\mathrm{dA}$ & $0.31+/-0.002$ & $0.11+/-0.003$ & $0.32+/-0.002$ & $0.066+/-0.001$ & $0.049+/-0.0009$ & $0.30+/-0.004$ & 0.85 \\
\hline
\end{tabular}

${ }^{a}$ All yields $(Y)$ are $[\mathrm{g} / \mathrm{g}]$. Glucose $(\mathrm{S})$, biomass $(\mathrm{X})$, ethanol $(\mathrm{E})$, glycerol $(\mathrm{G})$, acetate $(\mathrm{A})$, carbon dioxide $(\mathrm{CO} 2)$.

${ }^{\mathrm{b}} \mathrm{Strain}$ abbreviations as in Figure 1.

and easily folded, thereby minimally taxing the translation and folding machinery. WA did increase two-fold in ATP consumption, most likely because $\alpha$-amylase is 10-fold larger and likely more difficult to fold and has more disulfide bond pairing possibilities. In the $\Delta$ hac1 background, folding efficiency is likely decreased due to ER dysfunction. With native secretion, $d N$ did not require higher ATP maintenance consumption compared to WT. However, even the smaller, easier to fold IP resulted in ER stress that required significant ATP consumption compared to WT. dA, which was already stressed under WT, continued to show high ATP consumption. Despite the increased ATP consumption in dI and $\mathrm{dA}$, little protein was secreted.

\section{Transcription factors controlling oxidative stress, amino acid salvaging, and expression repression are linked to secretory response}

Growth phase transcriptomics measurements were carried out to identify cellular processes that were activated under the stresses of $H A C 1$ deletion and recombinant protein production. HAC1 deletion resulted in 339 significantly changed genes in the no recombinant protein case (WN vs. $\mathrm{dN}) . H A C 1$ deletions in the insulin strain and $\alpha$-amylase strain resulted in much larger cellular responses of 1628 (WI vs. dI) and 1511 (WA vs. dA) significantly expressed genes, respectively. KAR2 (ER chaperone) expression was significantly reduced upon $H A C 1$ deletion ( $\downarrow$ three-fold $\mathrm{dN}$ vs WTN, $P=1 \times 10^{-4}$ ) and the four yeast protein disulfide isomerases (PDI1, EUG1, MPD1, MPD2) reduced an average of 2.9 -fold $(P<0.05)$.

The effects of producing IP or $\alpha$-amylase within a strain background (WT or $H A C 1$ ) were not as pronounced as the effect of HAC1 deletion, 40 and 194 genes were significantly changed in WI (compared to WN) and WA (compared to WN). Likwsise, 74 and 90 genes were significantly changed for dI (compared to dN) and $\mathrm{dA}$ (compared to $\mathrm{dN}$ ).

To reduce the dimensionality of the data and identify putative TFs involved in protein secretion, the Reporter Transcription Factor algorithm was used [23]. TFs were scored by the modulation in expression level of genes that the TFs bind in the upstream region according to ChIP-chip data [24]. Therefore, the score is not indicative of change in the TF expression level itself, but of the genes under its influence. Reporter TF algorithm is useful, because although the statistical significance of an individual gene may not meet an arbitrary threshold, if several genes linked to the same TF have similar behavior, the likelihood of observing the group of genes is low, making TF identification very sensitive. Figure 3 shows significant secretory process TFs shown to be involved in up- and down-regulating different cellular process under their control. Interestingly, different TFs were identified for the two different proteins. This is likely the combined effect of different protein size and number of disulfide bonds. A complete list of significant transcription factors is provided in Additional files 5 and 6.

In WT (Figure 3a), several TFs were activated by protein secretion. Oxidative and osmotic stress pathway upregulation was common to both proteins. Oxidative stress is likely caused by ROS that is formed when Ero1p shuttles electrons to oxygen in disulfide bond formation [25]. Osmotic stress response, particular hypo-osmotic stress, strengthens the cell wall to counteract internal turgor pressure by changing the cell wall composition. This change in composition requires remodeling the secretory pathway by changing which components are trafficked to the cell wall [26]. Surprisingly, the Reporter TF algorithm found several Hac1p-influenced genes down-regulated. Genes that Hac1p binds from the ChIPchip data that are significantly down-regulated are KEG1, MCD4, and ERJ5. KEG1 and MCD4 genes are involved in glycan modifications and ERJ5 is a secondary ER chaperone [27-29]. These genes may be influenced by other TFs not included in the ChIP-chip network. Genes known to be regulated by Hac1p (KAR2 and ERO1) were not significantly changed upon secreting recombinant protein, indicating that there is not an actual Hac1p response in the WT.

Clear differences between large and small protein secretion emerge in WT. IP stimulated modification of the TGN through MCM1 and STE12. Overall expression 


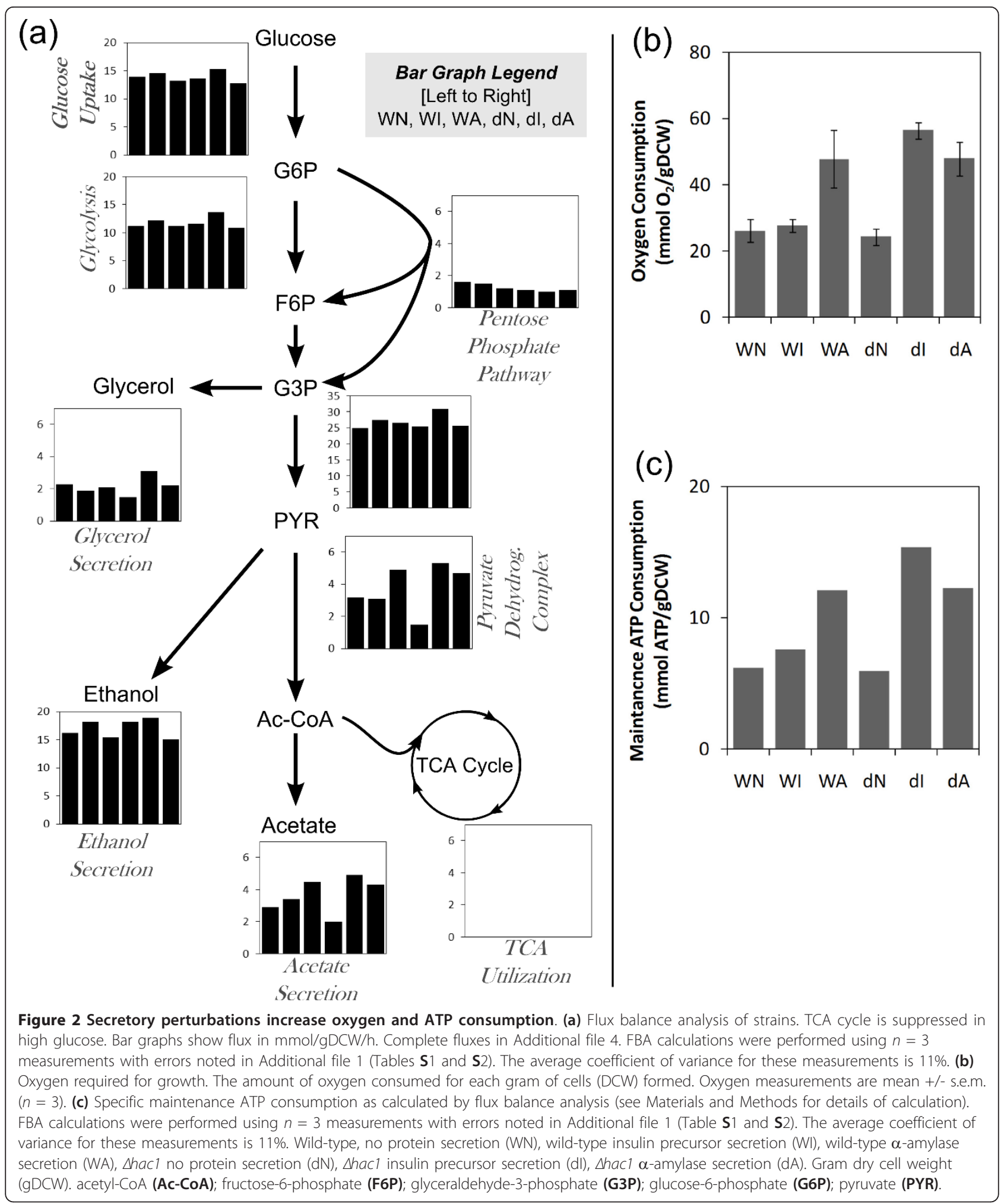

is reduced by altering mRNA degradation pathways via STO1. $\alpha$-amylase had a much larger effect on the cell, as compared to IP, as was implied by physiological parameters of Figure 1 and number of altered genes.
Additional oxidative and osmotic stress pathways were activated in WA, as well as a down-regulation in some amino acid synthesis pathways and overall reduction of transcription. 


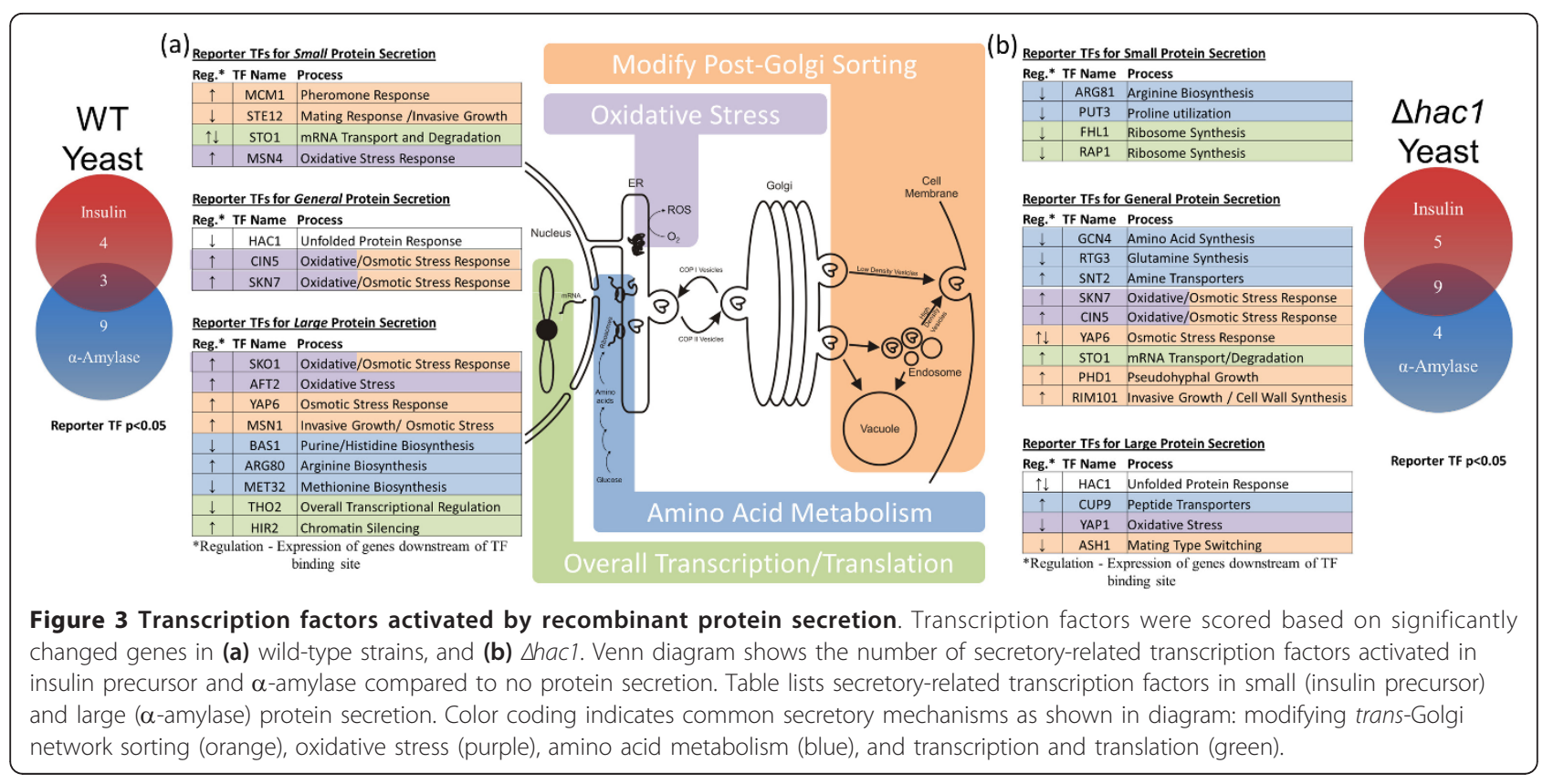

In the $\Delta$ hac1 background (Figure $3 \mathrm{~b}$ ), many of the effects found in WA, have become common to both IP and $\alpha$-amylase producing strains. HAC1 deletion clearly makes the cell more susceptible to recombinant secretion overload. Both insulin and $\alpha$-amylase secretion cause considerable oxidative stress response and downregulation of amino acid synthesis, including the general amino acid synthesis TF, Gcn4p. In dI, translational capacity repression is also employed (via Fhlp/Rap1p) and adjustments in amino acid metabolism. dA shows a mix of up- and down-regulation of genes that are controlled by Hac1p. Other TFs appear to be controlling these genes in the absence of $H A C 1$. Some oxidative and osmotic stress pathways appear independent of $H A C 1$. Skn7p and Cin5p were similarly activated in both WT and $\Delta$ hac1. Oxidative and hypo-osmotic stress, while important for managing the secretory pathway, appears not to be directly managed through the UPR.

\section{Thermodynamic irreversibilities in redox reactions can explain increased oxidative stress in slow protein folding conditions}

The increases in oxidative stress, oxygen consumption, and reduced growth observed in the study can be explained by electron transfer in ER redox pathways. Disulfide bond formation has been established to consume oxygen and produce ROS (and thereby consume cellular resources to protect against the ROS) in stoichiometric quantities with the number of disulfide bonds formed [9]. When non-native disulfide linkages are formed, these linkages must be rearranged to the correct disulfide pairings for the native protein to be folded, a process called disulfide isomerization [30].

Disulfide isomerization involves (a) breaking the nonnative bond by transferring electrons to the non-native bond creating a cysteine linkage with the PDI, and (b) creating a new disulfide linkage in the nascent protein by transferring the electrons to break the PDI-nascent protein linkage. By random pairing, the native disulfide bonds are found.

Directionality in these redox reactions is determined by thermodynamic favorability through electron affinity of the potential disulfide bonds. Disulfide isomerization is redox neutral, not requiring electron donors or acceptors. However, it does require each disulfide pairing to have a lower electron affinity than the next (non-native disulfide in folding protein < PDI-folding protein disulfide $<$ native disulfide in folding protein) to allow the electrons to transfer. Under slow folding conditions, PDI may hold the disulfide bond (oxidized state) for extended time because a native disulfide cannot be found, resulting in PDI being reduced by other moieties, likely GSH.

Given the observations in our experiments, and the thermodynamic reasoning immediately above, we propose a simple thermodynamic model of disulfide bond formation and breaking that explains increased oxidative stress, oxygen consumption, and reduced growth observed in our experiments. This model expands upon the mechanism by Cuozzo and Kaiser [13]. The thermodynamic model assumes there are PDI disulfide bonds that have electron affinities above and below the nascent 


\section{(A) Disulfide Bond Futile Cycle}

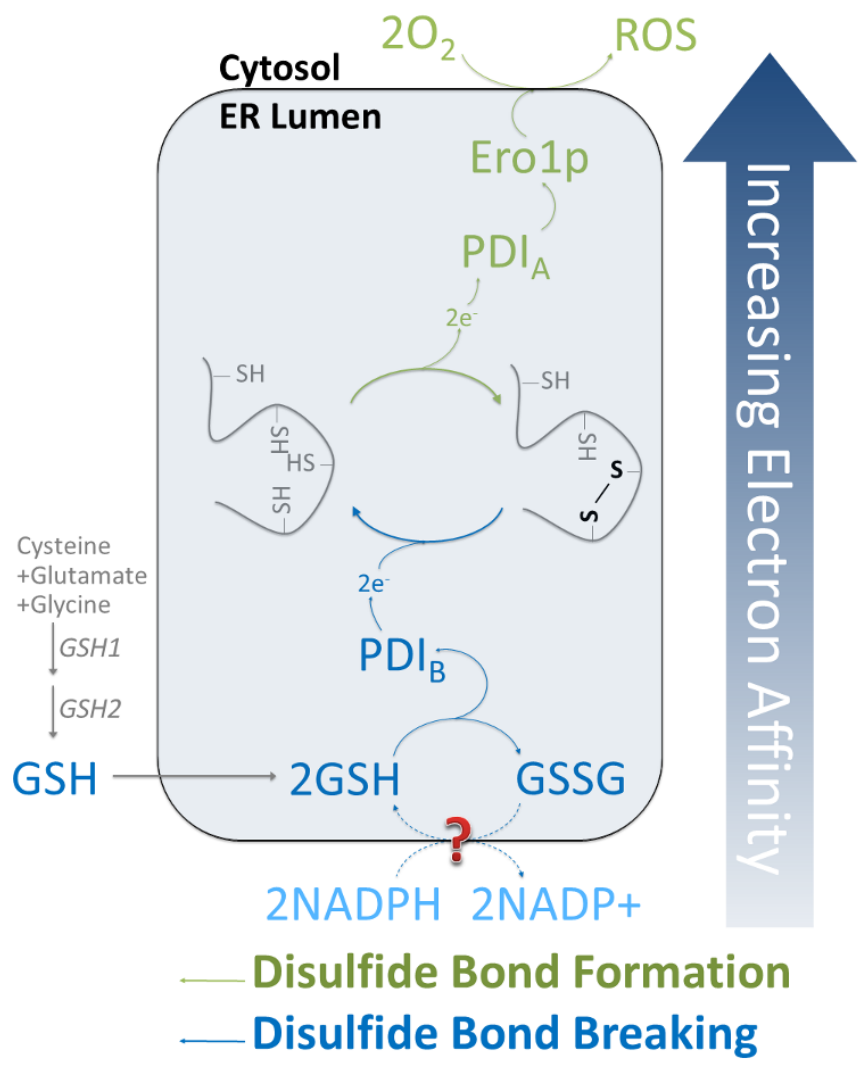

(B) Thermodynamic Model

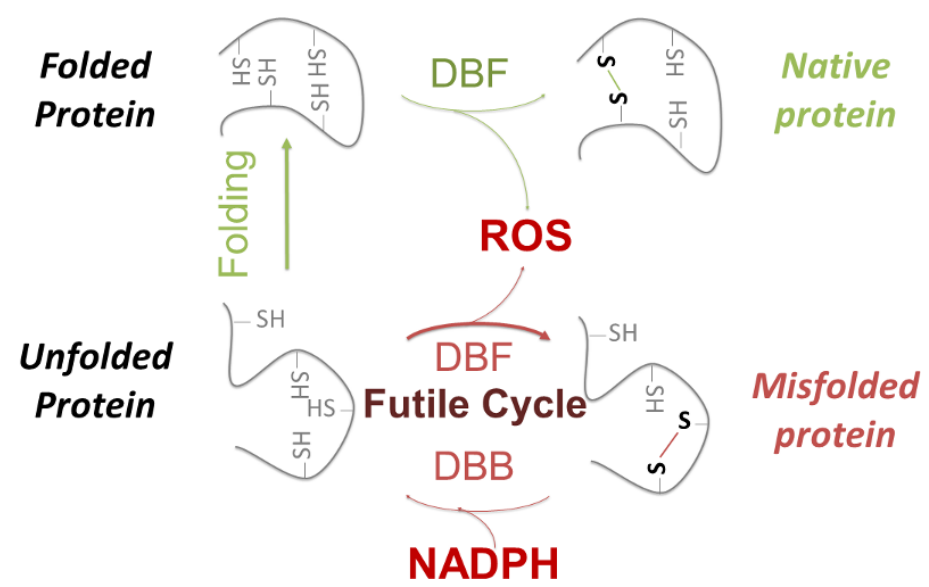

Figure 4 Proposed thermodynamic model predicts non-stoichiometric reactive oxygen species produced with incorrect disulfide bond formation. (a) In the model, forming and breaking an incorrect disulfide bond uses two protein disulfide isomerases (PDIs), one with electron affinity higher $\left(\left.P D\right|_{A}\right)$ and one lower $\left(\left.P D\right|_{B}\right)$ than the incorrect disulfide bond. In the formation phase, electrons are shuttled to molecular oxygen, resulting in ROS formation. In the breaking phase, electrons are passed from NADPH, through glutathione, to the protein. In both cases, electrons move along the electron affinity gradient. The net result is a futile cycle that is required to fix incorrect disulfide bonds, but expends redox energy. (b) The thermodynamic model predicts at fast folding rates near stoichiometric ROS is generated per disulfide bond formed. However, when folding rates are slow, the unfolded protein may go through many futile cycles, resulting in excess ROS. Glutathione (GSH), oxidized glutathione (GSSG), disulfide bond formation (DBF), disulfide bond breaking (DBB). 
proteins disulfide bonds (Figure 4a). The disulfide is formed by the typical oxidation pathway (Figure 4a, green) catalyzed by high electron affinity PDI (called $\mathrm{PDI}_{\mathrm{A}}$ here). Instead of isomerization, the incorrect disulfide is reduced by an electron donor with a low electron affinity (most likely a different PDI paralogue, called $\mathrm{PDI}_{\mathrm{B}}$ here) (Figure 4a, blue). The difference in electron affinity between the folding protein's cysteines and a specific PDI's cysteines can only allow the electrons to flow in one direction (toward the higher electron affinity cysteines) (Figure 4a). Therefore, a different PDI is required to form and break the incorrect disulfide bond. This futile cycle relies on a strong electron affinity gradient to complete an isomerization-like process. The net result of the futile cycle is GSH consumption and ROS production. This model implies that the ROS produced is not stoichiometrically linked to the number of disulfide bonds formed, but varies by the number of futile cycles before the correct bond is formed.

The metabolic and transcriptional data supports this model. Upon HAC1 deletion, ER chaperones (KAR2) and PDIs (PDI1, FUG1, MPD1, and MPD2) expression is reduced. This downregulation of ER chaperones and PDIs results in suppressed ER folding and disulfide bond formation in the $\Delta h a c 1$ mutants. In the $\mathrm{dN}$ case, minimal oxidation stress is seen. However, when there is an increased demand for protein folding and disulfide bond formation, as is the case for $\mathrm{dI}$ and dA case, we see high oxygen consumption, ATP requirements, and many oxidative stress pathways being activated transcriptionally. Although both folding and disulfide bond formation is down, an imbalance toward faster disulfide bond formation compared to folding will result in futile cycles. Therefore, this disulfide/folding imbalance acts as a catalyst for drastically increasing ROS production.

Based on this thermodynamic model, the relative rates of protein folding and disulfide bond formation for nascent peptides have important consequences for oxidative stress (Figure $4 \mathrm{~b}$ ). When folding is faster than disulfide bond formation, ROS is produced in near one-to-one amounts with the disulfide bonds formed. Under these conditions, isomerization may be more efficient to resort incorrect disulfide bonds, as native structures with low electron affinity disulfide pairs are favored, and isomerization does not produce ROS. However, when folding is slow compared to disulfide bond formation, as is the case when the protein folding machinery gets overloaded, the nascent peptides cycles through the futile redox cycle producing ROS and consuming GSH in excess to the final number of disulfide bonds formed. The physiological result of a high disulfide bond formation to ER folding rate is oxidative damage to a broad range of cellular proteins and consumption of reducing equivalents that could otherwise be used for anabolism.

\section{Discussion}

In this study, we have identified biological mechanisms related to protein synthesis and secretion by introducing perturbations to the cell, in the form of $H A C 1$ deletion and different recombinant protein expression, and measuring the system level cellular responses, via transcriptomics and metabolic fluxes. These measurements, combined with data analysis algorithms, Reporter TF algorithm and FBA, were able to identify cellular adjustments in (a) overall expression level, (b) post-Golgi sorting, (c) amino acid biosynthesis and savaging, and (d) oxidative stress. These biological effects are a result of the combined influence of protein synthesis and trafficking through the secretory pathway.

Overall transcription and translation were repressed in response to $\alpha$-amylase expression (a larger protein) and in the $\Delta h a c 1$ strains with any recombinant protein secretion. Repressing overall expression is a broad spectrum response used to adjust the rates of all other cellular processes to match the reduced folding capacity in the ER. Several mechanisms were used to alter overall expression: repressing mRNA synthesis, increasing mRNA degradation rates, and repressing protein translation rates through reducing ribosome numbers. Specifically, mRNA concentrations are lowered by decreasing RNA polymerase accessibility (HIR2), inhibiting transcriptional elongation (THO2), and controlling RNA degradation (STO1) [31,32]. Ribosome concentration, and thereby translation rates, can be reduced by the TFs Fhl1p and Rap1p which control expression of rRNA and ribosomal proteins [33]. This is seen in IP production in $\Delta$ hac1 strain, both by the reporter TFs (Figure 3) and by expression of ribosomal proteins (Additional file 7). In this context, extrachromosomal plasmids offer advantages over chromosomal expression. HIR2, whose mechanism is to silence the chromosome, would not affect extrachromosomal plasmids. Increased recombinant protein secretion would be accomplished by silencing native ER genes, while recombinant, plasmid-born gene would not be affected.

Pronounced adjustments to the TGN were observed in the transcriptome in all conditions. TFs involved in pheromone responses (STE12, MCM1, ASH1), invasive/pseudohyphal growth (STE12, MSN1, PHD1, RIM101), and osmotic stress (CIN5, SKN7, SKO1, YAP6, MSN1) were all identified by the Reporter TF algorithm and point to an underlying set of activities that are required to increase the traffic of secretory vesicles to the membrane. Invasive, pseudohyphal, and filamentous growth morphologies have a high surface to volume ratio and inherently require higher Golgi-to-cell membrane trafficking rates to supply cell membrane and cell wall components for growth. These altered morphologies can be activated through the filamentous and invasive response elements (FREs) [34] 
bound by STE12 and used to regulate PHD1 [35]. HAC1 deletion has been shown to cause filamentous growth [36].

Osmotic stress TFs are also responsible for affecting protein secretion, as the external cell wall must be strengthened in response to hypo-osmotic conditions, thereby requiring an efficient secretory pathway to ferry cell wall proteins [26]. MSN1 is known to induce starch degradation, requiring the actions necessary to secrete the appropriate enzymes through filamentous growth activation [37]. SKN7 has a dual role in invasive growth and osmotic stress [38]. Although osmotic stress TFs are commonly associated with the hyper-osmotic glycerol (HOG) pathway, Ypd1p can phosphorylate Skn7p, signaling the hypo-osmotic stress pathway [39]. Because there were no apparent hypo-osmotic conditions in this study, this indicates that these TFs are not directly controlled by osmotic conditions, but possibly through a secondary response to upregulation and increased secretion of cell wall proteins.

TGN TFs and/or the genes they regulate are possible targets for increasing Golgi-to-cell membrane trafficking. In S. cerevisiae, recombinant protein intended for secretion has been found mis-trafficked to the vacuole. This has been shown for insulin and green fluorescent protein secretion in yeast $[40,41]$. Proteins involved in vesicle trafficking, namely Sly1 and Munc18 have been found to increase recombinant secretory rates in Chinese hamster ovarian ( $\mathrm{CHO}$ ) and several mammalian cell lines $[42,43]$. It is likely that similar proteins are present in yeast and could be exploited for improving protein secretion.

Significant alterations in amino acid metabolism were observed, particularly in the $\triangle$ hac1 strains. De novo amino acid synthesis (GCN4, BAS1, MET32, ARG81, $R T G 3)$ was suppressed. On the surface, this appears contradictory, as increased amino acid requirements should be observed with recombinant protein production. However, this decrease in de novo amino acid synthesis is accompanied by observed increases in scavenging mechanisms for amino acids (SNT2, CUP9, PUT3). High scavenging rates and decrease synthesis imply high protein degradation rates where the degraded proteins result in available amino acids for scavenging; reducing the need for newly synthesized amino acids. This is consistent with either ERAD, a process where proteins that are stalled in the ER are transported back into the cytoplasm for degradation by the proteosome, or vacuolar-localized protein degradation. In either case, the cell is expending energy on synthesizing proteins that are ultimately degraded. These effects appear in the strains that are the slowest growing with the highest ATP requirements (Figures $1 \mathrm{~b}$ and $2 \mathrm{~b}$ ). In these cases the ER folding capacity is likely saturated, resulting in ER holdup and ERAD.
Oxidative stress TFs were also found in all conditions. Several were dual oxidative/osmotic stress TFs (CIN5, $S K N 7, S K O 1)$, and others were dedicated to oxidative stress only (AFT2, YAP1). TFs were found in all three of the major oxidative stress signaling pathways, (a) the Hog1 MAPK pathway (where SKO1 is the DNA binding agent), (b) Sln1 pathway (where SKN7 is the DNA binding agent), and (c) YAP1 and CIN5, which directly sense oxidative stress and bind DNA [44]. The cell's control machinery appears to have hard-wired oxidative stress responses to increased secretory demand, as oxidative/ hypo-osmotic pathways have a high degree of overlap, which is appropriate because increased secretion of cell wall proteins will result in higher oxidative stress. In particular, Skn7p, which has already been mentioned for its role in managing secretory pathway directly in an osmotic stress pathway, can also activate oxidative stress response genes [45].

Oxidative stress was pronounced with all secretory perturbations and has been identified in other studies to be associated with secretory stress $[1,17]$. Futile cycling may be the dominant disulfide resorting pathway when folding is limited. In previous studies, oxidative stress, induced by tunicamycin, a N-linked glycosylation inhibitor, increased with ER stress, despite no increase in the net disulfide bond formation demand [17]. The futile cycle does predict non-stoichiometric ROS formation, while isomerization does not. ROS can be formed at potentially limitless amounts through multiple rounds of disulfide formation and breaking. This will occur under conditions where the rate of folding is slow, a result of proteins that are specifically difficult to fold, or a result of the overall ER folding capacity being saturated. As well, futile cycling will increase as the number of available cysteine residues available for disulfide bonding increase, as is the case for $\alpha$-amylase, due to the extended amount of isomerization that may be needed to form the correct disulfide bonds.

One implication of the proposed thermodynamic model is that PDI paralogues, or cysteines within a PDI, must exist at different electron affinities that are above and below the electron affinity of the protein to be folded. Although in vivo redox potentials of PDI cysteine pairs were not measured, from first principles it would appear highly likely that these PDIs would need different redox potentials to carry out isomerization. In Figure 4a, we assume that only PDIs interact with the folding protein. This appears the case, as kinetic rates for direct glutathione oxidation/reduction are too slow to be physiologically relevant [9]. Electron affinity (and therefore redox potential) is broadly determined by the proximity of the two cysteines, with the proximity determined by the current structure of the protein [46]. Cysteines that are in the correct orientation will have a low electron 
affinity and easily form disulfide bonds, while cysteines that are not in the correct orientation will have a high electron affinity and will have unstable disulfide bonds. Therefore, the electron affinity of a correctly folded/correct disulfide bond would be lower than that of a misfolded or incorrect disulfide bond. This difference in electron affinity may allow PDIs to selectively break disulfides with high electron affinity (incorrect bonds), but not disulfide bonds with low affinity (correct bonds).

The need for different PDIs to form or break disulfide bonds may explain the need for many PDI homologues in the ER, each with different structures, and therefore different electron affinities. These PDIs can only span a finite range of electron affinities, and there may be implications for proteins that have disulfide pairs with electron affinities higher than the highest PDI or lower than the lowest PDI. If no PDI has a lower electron affinity than an incorrect disulfide bond, then the disulfide bond cannot be broken and the protein is terminally misfolded. As well, a protein that has a native disulfide pairing with an electron affinity higher than any PDI cannot form a bond. This may be the case when recombinant proteins are being processed in the ER.

Futile cycling as a large potential ROS source has broad implications on the cell. Tu and Weissman predict Erolpproduced ROS that is one-to-one with disulfide bond formation could attribute approximately $25 \%$ of cellular ROS to the secretory pathway [1]. Therefore, even larger ROS production is likely if the futile cycle is the dominant disulfide resorting pathway under folding stress. This also has implications on GSH and possibly NADPH availability, as it is doubly consumed (a) by the reduction of ROS and (b) directly in the futile cycle. The futile cycle limits reducing equivalents needed for anabolic processes, and may explain the reduced growth rates observed in folding stressed strains (WA, dI, and dA).

In all, Figure $4 \mathrm{~b}$ highlights that the relative rates of two processes, protein folding and disulfide bond formation, must be kept in balance to avoid significant cellular stress. If disulfide bond formation is fast compared to folding, high futile cycle use will result in high ROS formation, NADPH loss, and high protein degradation as a result of ERAD. This scenario is observed in the $\Delta$ hac1 strains $\mathrm{dI}$ and $\mathrm{dA}$.

The engineering implications for protein secretion become much clearer with this understanding of protein folding to disulfide bond formation ratio. When overexpressing a recombinant protein, an optimal expression must be found, where transcription is as high as possible without overloading the ER folding capacity and sending the cell into an oxidative stressed state. This optimal expression level will be different for different proteins, as protein folding rates will vary according to the protein size and structure. We see this in comparing IP and $\alpha$-amylase expression. The concept of an optimal expression has been identified heuristically, in the present study we identify the competing molecular effects that could define these phenomena [47]. This optimal expression ratio extends to recombinant proteins that do not have disulfide bonds. For recombinant proteins without disulfide bonds, recombinant protein folding in the ER will consume folding resources, thus slowing down folding rates. Although the recombinant protein has no disulfide bonds, many native proteins still require disulfide bonds. Because of this, the folding to disulfide bond formation ratio will be disturbed, resulting in similar ROS stress.

To maintain an optimal ratio, either protein folding rates must increase or oxidation rates decrease. Overexpression of chaperones that increase folding capacity has successfully been used to increase protein secretion $[6,48]$. For particularly large or difficult to fold proteins this may not be adequate. A new approach may be to limit the oxidation rate of Erolp to slow down the first step of the futile cycle. This would be done in concert with repressing ERAD, as proteins would have long retention times in the ER. In this scenario, recombinant proteins would be slowly folded, albeit without high cellular stresses. This would result in longer overall process times, but may be required for difficult to fold proteins.

\section{Conclusion}

In this study, we identified post-Golgi vesicle sorting, high protein degradation rates, repressed overall expression, and oxidative stress in response to +/- UPR strains secreting different sized recombinant protein. These processes were identified through scoring TFs and estimating alteration to the metabolic network. These observations imply our proposed futile cycling is the dominant disulfide resorting pathway in the ER and explains non-stoichiometric ROS formation seen in our study and elsewhere. The futile cycle model, producing ROS and consuming GSH, has a clear thermodynamic driving force compared to disulfide bond isomerization. If correct, futile cycling is likely the dominant mechanism under secretory stress. This interplay between protein folding and futile cycling sheds light on a largely empirical understanding of engineering protein secretion and implies the relative rates of protein folding and disulfide bond formation are critical to maintaining cellular homeostasis. This increased molecular understanding of the secretory pathway should allow for more insightful design of secretory engineering strategies.

\section{Methods}

\section{Strains and media}

All experiments were performed in the background of CEN.PK 113-5D (MAT a SUC2 MAL2-8 ${ }^{\mathrm{C}}$ ura3-52, P. 
Kötter, Frankfurt, Germany) [49]. Genomic DNA from Y05650 (BY4741; Mat a; his3D1; leu2D0; met15D0; ura3D0; YFL031w::kanMX4, obtained from EUROSCARF) was used as a template for the HAC1 knockout cassette. Standard molecular biology techniques were used [50] and all plasmids were maintained in Escherichia coli DH5 $\alpha$ in Luria Bertani (LB) broth with $80 \mathrm{mg} / \mathrm{L}$ ampicillin. PCR primers are listed in Additional file 8.

\section{Cloning}

Genomic DNA was purified from Y05650 using Fast DNA Spin Kit for Soil (MP Biomedicals Solon, OH, USA). A $2.6 \mathrm{~kb}$ DNA fragment containing the genomic replacement of $H A C 1$ with KanMX and flanking regions was amplified by PCR using primers KT007/KT008 (Additional file 8). The HAC1::kanMX4 fragment was integrated at the HAC1 loci of CEN.PK 113-5D by standard yeast transformation [51] and selected on $200 \mathrm{mg} /$ L G418 to create the $\Delta$ hac1 strain. Correct integration was confirmed by PCR.

DNA coding for an insulin precursor with a Yap3 preleader sequence and the TA57 pro-leader sequence and spacers as described [18] for correct secretory processing was synthesized with optimal codon usage for yeast and delivered on plasmid pUC57-Yap3Insulin (GenScript Co. Piscataway, NJ, USA) (Additional file 9 for sequence). $\alpha$ Amylase DNA was amplified from Saccharomyces kluyveri YKM37 [52] using LZH018 and LZH039. The pre-pro-leader was amplified from pUC57-Yap3Insulin using primers LZH015 and LZH016. The pre-pro-leader was connected to the $\alpha$-amylase by fusion PCR of the two segments together using primers LZH015 and LZH039 [53]. The pre-pro-insulin and pre-pro-amylase were cloned into the SpeI/Sall or SpeI/EcoRI sites of p426GPD, respectively, downstream of the constitutive GAPDH promoter [54], to create pYapIns and pYapAmy. Plasmids p426GPD, pYapIns, and pYapAmy were transformed into CEN.PK 113$5 \mathrm{D}$ and $\Delta$ hac1 strains by standard methods [51].

\section{Fermentor conditions}

Strains were grown in SD-2xSCAA [55], containing $20 \mathrm{~g} / \mathrm{L}$ glucose, $6.7 \mathrm{~g} / \mathrm{L}$ yeast nitrogen base minus amino acids (Formedium, Norfolk, UK), $2 \mathrm{~g} / \mathrm{L} \mathrm{KH_{2 }} \mathrm{PO}_{4}(\mathrm{pH}=6$ by $\mathrm{NaOH}), 190 \mathrm{mg} / \mathrm{L}$ Arg, $108 \mathrm{mg} / \mathrm{L}$ Met, $52 \mathrm{mg} / \mathrm{L}$ Tyr, 290 mg/L Ile, 440 mg/L Lys, 200 mg/L Phe, $1260 \mathrm{mg} / \mathrm{L}$ Glu, $400 \mathrm{mg} / \mathrm{L} \mathrm{Asp}, 380 \mathrm{mg} / \mathrm{L} \mathrm{Val}, 220 \mathrm{mg} / \mathrm{L} \mathrm{Thr}, 130 \mathrm{mg} / \mathrm{L}$ Gly, 400 mg/L Leu, 40 mg/L Trp, 140 mg/L His, 1 g/L bovine serum albumin. Five hundred $\mathrm{mL}$ of medium was inoculated in a $1 \mathrm{~L}$ bioreactor (DasGip, Jülich, Germany) at $30^{\circ} \mathrm{C}, 600 \mathrm{rpm}$ agitation, 30 standard L/h air flow, $\mathrm{pH}$ controlled at 6 by $\mathrm{KOH}(2 \mathrm{M})$. Strains were inoculated to an $\mathrm{A}_{600}=0.01$ from late exponential phase cultures and $\mathrm{A}_{600}$ was measured throughout the cultivation. Dry cell weight $(\mathrm{DCW})$ was measured by filtering $5 \mathrm{~mL}$ of culture broth through a $0.45 \mu \mathrm{m}$ nitrocellulose filter and measuring the increased weight of the dry filter. Glucose, ethanol, glycerol, and acetate were measured using a Summit HPLC (Dionex, Thermo Scientific, Waltham, MA, USA) with an Aminex HPX-87H column (Bio-Rad, Hercules, CA, USA). Carbon dioxide and oxygen levels were measured in the off-gas and dissolved oxygen was monitored. Transcriptome samples were taken after 5+ doublings at $\mathrm{A}_{600}=1.0-1.4$. Triplicate fermentations were carried out for each strain.

\section{Protein quantification}

Insulin was measured by a modification of the assay by Snell et al. [56]. One $\mathrm{mL}$ of cell culture was centrifuged at $4000 \times \mathrm{g}$ for $4 \mathrm{~min}$. Eight parts supernatant was added to one part $0.1 \mathrm{~N} \mathrm{HCl}$ and $5.5 \mu \mathrm{M}$ sodium azide and stored at $4^{\circ} \mathrm{C}$ until measurement. Insulin concentration was determined by HPLC using a Luna $5 \mu \mathrm{C} 18(2)$ $(250 \mathrm{~mm} \times 4.6 \mathrm{~mm})$ (Phenomenex, Torrance, CA, USA) column and gradient-based elution. Buffer A contained $68 \mathrm{mM}$ phosphoric acid, $0.2 \mathrm{M}$ sodiumsulphate and $10 \%$ $(\mathrm{w} / \mathrm{v})$ acetonitrile in water, and Buffer B contained 50\% acetonitrile in water. HPLC was run with $25 \mu \mathrm{L}$ injections at $1 \mathrm{~mL} / \mathrm{min}$ and $50^{\circ} \mathrm{C}$. Gradient protocol: $20 \% \mathrm{~B}$ for 10 min. Linear gradient from $20 \%$ B to $60 \%$ B over $10 \mathrm{~min}$. Hold at $60 \%$ B for $5 \mathrm{~min}$ and then to $20 \%$ B for $3 \mathrm{~min}$ to re-equilibrate for next sample. Insulin standards eluted at $22.6 \mathrm{~min}$ and insulin precursor at 20.0 min. HPLC peaks were verified to be the correct protein by SDS-PAGE. Human insulin was used as a standard (Sigma, St. Louis, MO, USA).

$\alpha$-amylase concentration was calculated from enzyme activity. $\alpha$-amylase activity was measured using the Ceralpha kit (Megazyme K-CERA, Wicklow, Ireland) using $\alpha$-amylase from Aspergillus oryzae (Sigma, St. Louis, MO, USA) as a standard. This conversion was calculated using a $1.79 \mathrm{U} / \mathrm{mg}$ (weight includes salts and purified protein) standard from Sigma using the Protein 80 chip on the Bioanalyzer (Agilent, Santa Clara, CA, USA). By this, $\alpha$-amylase was found to be $0.0257 \mathrm{~g} \alpha$-amylase $/ \mathrm{g}$ total. $\alpha$-amylase activity was then converted to mass using $70 \mathrm{U} / \mathrm{mg} \alpha$-amylase protein.

\section{Transcriptome analysis}

Samples for microarray were taken as described previously and stored at $-80^{\circ} \mathrm{C}$ until processing [57]. RNA was isolated using the RNeasy Minikit (Qiagen, Valencia, CA, USA). Cells were lysed in RNeasy RLT buffer using Lysing Matrix C (MP Biomedicals Solon, OH, USA) in a Fast Prep 24 (MP Biomedicals Solon, OH, USA) as follows: $20 \mathrm{~s}$ at speed $6,1 \mathrm{~min}$ at $4{ }^{\circ} \mathrm{C}, 20 \mathrm{~s}$ at speed 6. RNA was processed to aRNA using the Genechip 3' IVT Express Kit (Affymetrix, Santa Clara, CA, USA) and hybridized/scanned on the Yeast Genome 2.0 
Array (Affymetrix, Santa Clara, CA, USA) following commercial protocols to create CEL files.

Images were analyzed using R 2.10 .1 statistical software and the 'affy' and 'limma' packages as described previously [58]. Briefly, background normalization was carried out using robust multi-array (RMA) average method with perfect match (PM) probes only. Interchip normalization used the qspline algorithm with median polish summary method. Statistical analysis was carried out by comparison of triplicate bioreactor measurements for each strain. Emperical Bayesian statistics were used to moderate standard errors within each gene and Benjamini-Hochberg's method to adjust for multiple testing. Microarray data was submitted to the GEO database and have accession number GSE27062 (see http://www. ncbi.nlm.nih.gov/geo/query/acc.cgi?

token=dpyzfywysoqecbk\&acc=GSE27062).

\section{Reporter transcription factor analysis}

Transcription factor activity was scored using the Reporter Effector algorithm [23]. Transcription factorDNA interactions were gathered from ChIP-chip with $P$ $<0.001$ [24]. Significant interactions were found for 176 transcription factors regulating 3,796 genes for a total of 10,849 unique interactions. Gene $P$ values from comparing different strains were used to score transcription factors that were known to bind to the upstream DNA. Transcription factors with $P<0.05$ of being activated between conditions are reported.

\section{Flux balance analysis}

Estimates of intracellular reaction rates were performed using measured exchange fluxes of glucose, ethanol, acetate, glycerol, and carbon dioxide. Model-based error correction was used to close carbon and electron balances [59]. Flux balance analysis was carried out using a 85 reaction model of yeast central carbon metabolism and biomass yield were used [21]. Additional file 4 contains the complete results of the analysis which are used to estimate ATP consumption in the different strains.

\section{Additional material}

Additional file 1: Measured exchange fluxes in strains. Measured metabolite exchange fluxes for strains used in this study.

Additional file 2: Final glycerol concentration of WT and $\Delta$ hac 1 strains. Measured glycerol titers at end of fermentation for strains used in this study.

Additional file 3: Estimated exchange fluxes. Metabolite exchange fluxes as estimated by error-correction algorithm for strains in this study.

Additional file 4: Intracellular fluxes for metabolic network. Flux balance analysis estimates of internal fluxes for strains in thus study. Additional file 5: Reporter TFs for WT protein secretion. Transcription factors activated by recombinant protein secretion in wild-type background.
Additional file 6: Reporter TFs for $\Delta$ hac1 protein secretion

Transcription factors activated by recombinant protein secretion in $\Delta$ hacl background.

Additional file 7: Expression profiles for ribosomal proteins. mRNA concentrations for yeast ribosomal proteins as determined by DNA microarray.

Additional file 8: Oligonucleotides used in this study. PCR primers used for cloning and validation.

Additional file 9: Synthesized insulin precursor DNA sequence. DNA sequence for insulin precursor used in this study.

\section{Abbreviations}

ATP: adenosine triphosphate; $\mathrm{dA}$ : $\Delta$ hacl secreting a-amylase; dl: $\Delta$ hacl secreting IP; Dn: $\Delta$ hacl with empty vector; DNA: deoxyribonucleic acids; ER: endoplasmic reticulum; ERAD: ER-associated degradation; FBA: flux balance analysis; FRE: filamentous and invasive responsive elements; GSH: reduced glutatathione; HOG: hyper-osmotic glycerol; IP: insulin precursor; mRNA: messenger RNA; NADPH: nicotinamide adenine dinucleotide phosphate; PDI: protein disulfide isomerase; RNA: ribonucleic acid; ROS: reactive oxygen species; rRNA: ribosomal RNA; TF: transcription factor; TGN: trans-Golgi network; UPR: unfolded protein response; WA: WT secreting a-amylase; WI: WT secreting IP; WN: WT with empty vector; WT: wild-type.

\section{Acknowledgements}

We thank Dr Intawat Nookaew for assistance with transcriptome analysis and FBA. We thank NIH F32 Kirschstein NRSA fellowship (F32 GM083647), The Knut and Alice Wallenberg Foundation, EU Framework VII project SYSINBIO (Grant no. 212766), European Research Council project INSYSBIO (Grant no. 247013), and the Chalmers Foundation for funding.

\section{Author details}

'Department of Chemical and Biological Engineering, Chalmers University of Technology, Kemivägen 10, SE-41296 Göteborg, Sweden. ${ }^{2}$ Department of Chemical and Biological Engineering, Northwestern University, 2145 Sheridan Rd. Tech E136, Evanston, IL 60208, USA.

\section{Authors' contributions}

$\mathrm{KT}, \mathrm{DP}$, and JN designed the experiment. $\mathrm{KT}$ and $\mathrm{ZL}$ carried out all cloning, fermentations, and analytical measurements. $K T$ did primary calculations in transcriptomics and metabolic flux data. $\mathrm{KT}, \mathrm{ZL}, \mathrm{DP}$, and JN analyzed data and wrote the manuscript. DP and JN supervised the research. All authors have read and approved of the final manuscript.

\section{Competing interests}

The authors declare that they have no competing interests.

Received: 7 February 2012 Accepted: 1 March 2012

Published: 1 March 2012

\section{References}

1. Tu BP, Weissman JS: Oxidative protein folding in eukaryotes: mechanisms and consequences. Journal of Cell Biology 2004, 164(3):341-346.

2. Petranovic D, Tyo K, Vemuri GN, Nielsen J: Prospects of yeast systems biology for human health: integrating lipid, protein and energy metabolism. FEMS Yeast Res 2010, 10(8):1046-1059.

3. Idiris A, Tohda H, Kumagai H, Takegawa K: Engineering of protein secretion in yeast: strategies and impact on protein production. Applied Microbiology and Biotechnology 2010, 86(2):403-417.

4. Wedekind A, O'Malley MA, Niebauer RT, Robinson AS: Optimization of the human adenosine A(2)a receptor yields in Saccharomyces cerevisiae. Biotechnology Progress 2006, 22(5):1249-1255.

5. Valkonen M, Penttila M, Saloheimo M: Effects of inactivation and constitutive expression of the unfolded-protein response pathway on protein production in the yeast Saccharomyces cerevisiae. Applied and Environmental Microbiology 2003, 69(4):2065-2072. 
6. Shusta EV, Raines RT, Pluckthun A, Wittrup KD: Increasing the secretory capacity of Saccharomyces cerevisiae for production of single-chain antibody fragments. Nature Biotechnology 1998, 16(8):773-777.

7. Ellgaard L, Molinari M, Helenius A: Setting the standards: Quality control in the secretory pathway. Science 1999, 286(5446):1882-1888.

8. Bhamidipati A, Denic V, Quan EM, Weissman JS: Exploration of the topological requirements of ERAD identifies Yos9p as a lectin sensor of misfolded glycoproteins in the ER lumen. Molecular Cell 2005, 19(6):741-751.

9. Tu BP, Ho-Schleyer SC, Travers KJ, Weissman JS: Biochemical basis of oxidative protein folding in the endoplasmic reticulum. Science 2000, 290(5496):1571-1574

10. Frand AR, Cuozzo JW, Kaiser CA: Pathways for protein disulphide bond formation. Trends in Cell Biology 2000, 10(5):203-210.

11. Tu BP, Weissman JS: The FAD- and O-2-dependent reaction cycle of Ero1mediated oxidative protein folding in the endoplasmic reticulum. Molecular Cell 2002, 10(5):983-994.

12. Kulp MS, Frickel EM, Ellgaard L, Weissman JS: Domain architecture of protein-disulfide isomerase facilitates its dual role as an oxidase and an isomerase in Ero1p-mediated disulfide formation. Journal of Biological Chemistry 2006, 281(2):876-884

13. Cuozzo JW, Kaiser CA: Competition between glutathione and protein thiols for disulphide-bond formation. Nature Cell Biology 1999, 1(3):130-135.

14. Sevier CS, Kaiser CA: Ero1 and redox homeostasis in the endoplasmic reticulum. Biochimica Et Biophysica Acta-Molecular Cell Research 2008, 1783(4):549-556

15. Chakravarthi S, Jessop CE, Bulleid NJ: The role of glutathione in disulphide bond formation and endoplasmic-reticulum-generated oxidative stress. EMBO Rep 2006, 7(3):271-275.

16. Patil $C$, Walter $P$ : Intracellular signaling from the endoplasmic reticulum to the nucleus: the unfolded protein response in yeast and mammals. Current Opinion in Cell Biology 2001, 13(3):349-356.

17. Kimata Y, Ishiwata-Kimata Y, Yamada S, Kohno K: Yeast unfolded protein response pathway regulates expression of genes for anti-oxidative stress and for cell surface proteins. Genes to Cells 2006, 11(1):59-69.

18. Kjeldsen T, Pettersson AF, Hach M: The role of leaders in intracellular transport and secretion of the insulin precursor in the yeast Saccharomyces cerevisiae. Journal of Biotechnology 1999, 75(2-3):195-208.

19. Kjeldsen T: Yeast secretory expression of insulin precursors. Appl Microbiol Biotechnol 2000, 54(3):277-286.

20. Nagashima T, Yamamoto Y, Gomi K, Kitamoto K, Kumagai C: A novel culture method for high level production of heterologous protein in Saccharomyces cerevisiae. Biosci Biotechnol Biochem 1994, 58(7):1292-1296.

21. Forster J, Gombert AK, Nielsen J: A functional genomics approach using metabolomics and in silico pathway analysis. Biotechnology and Bioengineering 2002, 79(7):703-712.

22. Blank LM, Sauer U: TCA cycle activity in Saccharomyces cerevisiae is a function of the environmentally determined specific growth and glucose uptake rates. Microbiology 2004, 150(Pt 4):1085-1093.

23. Oliveira A, Patil K, Nielsen J: Architecture of transcriptional regulatory circuits is knitted over the topology of bio-molecular interaction networks. BMC Systems Biology 2008, 2(1):17.

24. Harbison CT, Gordon DB, Lee TI, Rinaldi NJ, Macisaac KD, Danford TW, Hannett NM, Tagne JB, Reynolds DB, Yoo J, et al: Transcriptional regulatory code of a eukaryotic genome. Nature 2004, 431(7004):99-104.

25. Riemer J, Bulleid N, Herrmann JM: Disulfide Formation in the ER and Mitochondria: Two Solutions to a Common Process. Science 2009, 324(5932):1284-1287.

26. Levin DE: Cell wall integrity signaling in Saccharomyces cerevisiae. Microbiology and Molecular Biology Reviews 2005, 69(2):262-+.

27. Carla Fama M, Raden D, Zacchi N, Lemos DR, Robinson AS, Silberstein S: The Saccharomyces cerevisiae YFR041C/ERJ5 gene encoding a type I membrane protein with a J domain is required to preserve the folding capacity of the endoplasmic reticulum. Biochimica Et Biophysica ActaMolecular Cell Research 2007, 1773(2):232-242.

28. Nakamata K, Kurita T, Bhuiyan MSA, Sato K, Noda Y, Yoda K: KEG1/YFR042w encodes a novel Kre6-binding endoplasmic reticulum membrane protein responsible for beta-1,6-glucan synthesis in Saccharomyces cerevisiae. Journal of Biological Chemistry 2007, 282(47):34315-34324.
29. Gaynor EC, Mondesert G, Grimme SJ, Reed SI, Orlean P, Emr SD: MCD4 encodes a conserved endoplasmic reticulum membrane protein essential for glycosylphosphatidylinositol anchor synthesis in yeast. Molecular Biology of the Cell 1999, 10(3):627-648.

30. Laboissière M, Sturley $S$, Raines R: The essential function of proteindisulfide isomerase is to unscramble non-native disulfide bonds. Journal of Biological Chemistry 1995, 270(47):28006-28009.

31. Piruat J, Aguilera A: A novel yeast gene, THO2, is involved in RNA pol II transcription and provides new evidence for transcriptional elongationassociated recombination. Embo Journal 1998, 17(16):4859-4872.

32. Das B, Butler JS, Sherman F: Degradation of normal mRNA in the nucleus of Saccharomyces cerevisiae. Molecular and Cellular Biology 2003, 23(16):5502-5515.

33. Rudra D, Mallick J, Zhao Y, Warner JR: Potential interface between ribosomal protein production and pre-rRNA processing. Molecular and Cellular Biology 2007, 27(13):4815-4824.

34. Madhani HD, Styles CA, Fink GR: MAP kinases with distinct inhibitory functions impart signaling specificity during yeast differentiation. Cell 1997, 91(5):673-684.

35. Erdman S, Lin L, Malczynski M, Snyder M: Pheromone-regulated genes required for yeast mating differentiation. Journal of Cell Biology 1998, 140(3):461-483.

36. Schroder M, Chang JS, Kaufman RJ: The unfolded protein response represses nitrogen-starvation induced developmental differentiation in yeast. Genes \& Development 2000, 14(23):2962-2975.

37. Lambrechts MG, Bauer FF, Marmur J, Pretorius IS: Muc1, a mucin-like protein that is regulated by Mss10, is critical for pseudohyphal differentiation in yeast. Proceedings of the National Academy of Sciences of the United States of America 1996, 93(16):8419-8424.

38. Palecek SP, Parikh AS, Kron SJ: Genetic analysis reveals that FLO11 upregulation and cell polarization independently regulate invasive growth in Saccharomyces cerevisiae. Genetics 2000, 156(3):1005-1023.

39. Tao W, Deschenes RJ, Fassler JS: Intracellular glycerol levels modulate the activity of $S \ln 1 p$, a Saccharomyces cerevisiae two-component regulator. Journal of Biological Chemistry 1999, 274(1):360-367.

40. Zhang BY, Chang A, Kjeldsen TB, Arvan P: Intracellular retention of newly synthesized insulin in yeast is caused by endoproteolytic processing in the golgi complex. Journal of Cell Biology 2001, 153(6):1187-1197.

41. Huang DG, Gore PR, Shusta EV: Increasing Yeast Secretion of Heterologous Proteins by Regulating Expression Rates and PostSecretory Loss. Biotechnology and Bioengineering 2008, 101(6):1264-1275.

42. Peng RW, Guetg C, Tigges M, Fussenegger M: The vesicle-trafficking protein munc18b increases the secretory capacity of mammalian cells. Metabolic Engineering 2010, 12(1):18-25.

43. Peng RW, Fussenegger M: Molecular Engineering of Exocytic Vesicle Traffic Enhances the Productivity of Chinese Hamster Ovary Cells. Biotechnology and Bioengineering 2009, 102(4):1170-1181.

44. Ikner A, Shiozaki K: Yeast signaling pathways in the oxidative stress response. Mutation Research-Fundamental and Molecular Mechanisms of Mutagenesis 2005, 569(1-2):13-27.

45. Li S, Dean S, Li ZJ, Horecka J, Deschenes RJ, Fassler JS: The eukaryotic twocomponent histidine kinase $\mathrm{S} \ln 1 \mathrm{p}$ regulates $\mathrm{OCH} 1$ via the transcription factor, Skn7p. Molecular Biology of the Cell 2002, 13(2):412-424.

46. Hatahet F, Ruddock LW: Protein Disulfide Isomerase: A Critical Evaluation of Its Function in Disulfide Bond Formation. Antioxidants \& Redox Signaling 2009, 11(11):2807-2850.

47. Wittrup K, Robinson A, Parekh R, Forrester K: Existence of an Optimum Expression Level for Secretion of Foreign Proteins in Yeast. Annals of the New York Academy of Sciences 1994, 745(1):321-330.

48. Robinson AS, Bockhaus JA, Voegler AC, Wittrup KD: Reduction of BiP levels decreases heterologous protein secretion in Saccharomyces cerevisiae. Journal of Biological Chemistry 1996, 271(17):10017-10022.

49. van Dijken JP, Bauer J, Brambilla L, Duboc P, Francois JM, Gancedo C, Giuseppin MLF, Heijnen JJ, Hoare M, Lange HC, et al: An interlaboratory comparison of physiological and genetic properties of four Saccharomyces cerevisiae strains. Enzyme and Microbial Technology 2000, 26(9-10):706-714.

50. Sambrook J, Fritsch EF, Maniatis T: Molecular Cloning: A Laboratory Manual. Cold Springs, NY, USA: Cold Spring Harbor Laboratory Press; 2 1989. 
51. Gietz RD, Woods RA: Transformation of yeast by lithium acetate/singlestranded carrier DNA/polyethylene glycol method. Guide to Yeast Genetics and Molecular and Cell Biology, Pt B 2002, 350:87-96.

52. Moller $\mathrm{K}$, Sharif MZ, Olsson L: Production of fungal alpha-amylase by Saccharomyces kluyveri in glucose-limited cultivations. J Biotechnol 2004, 111(3):311-318.

53. Pont-Kingdon G: Creation of Chimeric Juctions, Deletions, and insertions by PCR. In PCR protocols.. 2 edition. Edited by: Bartlett JMS, Stirling D. Totowa, NJ, USA: Humana Press Inc.; 2003:511-515.

54. Mumberg D, Müller R, Funk M: Yeast vectors for the controlled expression of heterologous proteins in different genetic backgrounds. Gene 1995, 156(1):119-122.

55. Wittrup KD, Benig V: Optimization of Amino-Acid Supplements for Heterologous Protein Secretion in Saccharomyces-Cerevisiae. Biotechnology Techniques 1994, 8(3):161-166.

56. Snel L, Damgaard U: Proinsulin heterogeneity in pigs. Horm Metab Res 1988, 20(8):476-480.

57. Usaite R, Patil KR, Grotkjaer T, Nielsen J, Regenberg B: Global transcriptional and physiological responses of Saccharomyces cerevisiae to ammonium, L-alanine, or L-glutamine limitation. Applied and Environmental Microbiology 2006, 72(9):6194-6203.

58. Salazar M, Vongsangnak W, Panagiotou G, Andersen MR, Nielsen J: Uncovering transcriptional regulation of glycerol metabolism in Aspergilli through genome-wide gene expression data analysis. Molecular Genetics and Genomics 2009, 282(6):571-586.

59. Stephanopoulos GN, Aristidou AA, Nielsen J: Material Balances and Data Consistency. Metabolic Engineering: Principles and Methodologies New York, New York: Academic Press; 1998.

doi:10.1186/1741-7007-10-16

Cite this article as: Tyo et al:: Imbalance of heterologous protein folding and disulfide bond formation rates yields runaway oxidative stress. $B M C$ Biology 2012 10:16.

\section{Submit your next manuscript to BioMed Central} and take full advantage of:

- Convenient online submission

- Thorough peer review

- No space constraints or color figure charges

- Immediate publication on acceptance

- Inclusion in PubMed, CAS, Scopus and Google Scholar

- Research which is freely available for redistribution 